\title{
Mantención de cerclaje luego de rotura prematura pretérmino de membranas antes de las 34 semanas se asocia a un aumento de corioamnionitis materna y mortalidad neonatal por sepsis
}

\author{
Claudia Celle T. ${ }^{1}$, Jorge A. Carvajal C. ${ }^{1} \mathrm{PhD}$. \\ ${ }^{1}$ Unidad de Medicina Materno-Fetal, División de Obstetricia y Ginecología, Facultad de Medicina, Pontificia Universidad \\ Católica de Chile.
}

\section{RESUMEN}

El cerclaje es un factor de riesgo de rotura prematura pretérmino de membranas (RPPM). La RPPM ocurre en aproximadamente un 38-65\% de los embarazos con cerclaje y no existe consenso aún sobre cuál es el manejo más adecuado: retirar o mantener el cerclaje. En esta revisión presentamos la evidencia disponible en relación al retiro versus mantención del cerclaje y su directa influencia sobre el resultado materno-perinatal, con el objetivo de proponer una pauta de manejo. La mantención del cerclaje se asoció a un aumento de la latencia al parto mayor a 48 horas, a un aumento en la incidencia de corioamnionitis clínica, y aumento en la incidencia de mortalidad neonatal por sepsis. Conclusión: En pacientes embarazadas con cerclaje que presentan RPPM antes de las 34 semanas proponemos como la conducta más adecuada la mantención del cerclaje sólo hasta completar la inducción de madurez pulmonar con corticoides, luego retirar el cerclaje y proceder al manejo habitual de un embarazo con RPPM antes de las 34 semanas.

\section{PALABRAS CLAVE: Rotura prematura pretérmino de membranas, cerclaje cervical}

\section{SUMMARY}

Cervical cerclage is a risk factor for preterm premature rupture of membranes (PPROM). PPROM occurs in about $38-65 \%$ of pregnancies with cerclage. There is no consensus on whether to remove or retain the cerclage after PPROM. Here we review the evidence about clinical management of PPROM in women with cerclage and its influence on maternal and neonatal outcome. Retained cerclage was found to be associated to a prolongation of pregnancy by more than 48 hours, to a higher incidence of maternal chorioamnionitis and to a higher incidence of neonatal mortality from sepsis. In pregnancies with cerclage complicated with PPROM before 34 weeks we propose to retain the cerclage just enough time to complete fetal lung maturation with corticosteroid therapy, then remove the cerclage and manage pregnancy as any PPROM before 34 weeks.

KEY WORDS: Preterm premature rupture of membranes, cervical cerclage 


\section{INTRODUCCIÓN}

Parto prematuro es aquel que ocurre antes de las 37 semanas de embarazo y es la principal causa de morbimortalidad neonatal (1). Es una patología altamente prevalente en nuestra población (5$6 \%$ y en el mundo entero $(12 \%)(1,2)$.

Una de las múltiples etiologías que explican la ocurrencia de parto prematuro es la incompetencia cervical, que se define como la incapacidad del cuello uterino para mantener el embarazo, en ausencia de contracciones uterinas (3). El tratamiento clásico de la incompetencia cervical ha sido el cerclaje cervical, que corresponde a la sutura circular en el cuello uterino, entre otras técnicas.

La utilidad del cerclaje cervical ha sido ampliamente demostrada para la prevención de parto prematuro en ciertos grupos de embarazos únicos: pacientes con antecedente de incompetencia cervical en embarazo previo o con antecedente de parto prematuro en embarazo previo y acortamiento cervical progresivo en el embarazo actual $(4,5)$.

La rotura prematura pretérmino de membranas (RPPM) es una causa frecuente de parto prematuro, responsable, aproximadamente, de un 30\% de los casos (6). En pacientes con cerclaje se ha descrito que la RPPM puede ocurrir hasta en un $38-65 \%$ de los embarazos (7).

La RPPM es, además, un factor de riesgo de morbilidad infecciosa materna y perinatal (6). En presencia de cerclaje, la morbilidad infecciosa asociada a RPPM podría aumentar, motivo por el cual la conducta general ha sido retirar el cerclaje una vez ocurrida la RPPM. Sin embargo, no existen estudios clínicos randomizados de buen diseño, que demuestren que la morbilidad infecciosa en embarazos que presentan RPPM asociada a la presencia de cerclaje disminuya al retirar el cerclaje. Por este motivo, no existe consenso en cuál es la conducta más apropiada: retirar o mantener el cerclaje en pacientes con RPPM.

Estudios que compararon embarazos con cerclaje que presentaban RPPM a embarazos en ausencia de cerclaje que presentaban RPPM $(8,9,10,11)$, no demostraron diferencias en la incidencia de morbilidad infecciosa materno-perinatal; a excepción de uno que demostró un aumento significativo de corioamnionitis clínica e histológica en embarazos con cerclaje, asociado a un aumento de la sepsis neonatal (9).

El objetivo de esta revisión es presentar la evidencia científica disponible en relación al retiro versus mantención del cerclaje en embarazos con RPPM antes de las 34 semanas, y evaluar su directa influencia sobre el resultado materno-perinatal. En base a los hallazgos nuestra intención es proponer una pauta de manejo para enfrentar a pacientes con cerclaje que presentan RPPM antes de las 34 semanas.

\section{MATERIAL Y MÉTODO}

Efectuamos una búsqueda en PubMed con los siguientes términos MeSH: "Cerclage" AND ("preterm premature rupture of the membranes" OR "PPROM" OR "Preterm PROM"). Incluimos estudios que evaluaran el manejo de embarazos con cerclaje que presentaran RPPM antes de las 34 semanas. Se recopiló información respecto de: método diagnóstico de RPPM, indicación de cerclaje y tipo de cerclaje (McDonald o Shirodkar), uso de corticoides, antibióticos y tocolíticos; latencia al parto, morbilidad infecciosa materna (corioamnionitis clínica y endometritis) y morbilimortalidad neonatal. Se excluyeron todos los estudios referentes a embarazos con RPPM sin cerclaje o a embarazos con cerclaje sin RPPM.

\section{RESULTADOS}

Resultados de la búsqueda: Arrojó 114 publicaciones. La lectura del título y resumen identificó 12 estudios que abordaban el tema de esta revisión. Luego de la lectura del texto completo de los 12 estudios, se excluyeron siete: cuatro que compararon embarazos con cerclaje que presentaban RPPM a embarazos con RPPM en ausencia de cerclaje $(8,9,10,11)$, dos revisiones sistemáticas $(12,13)$ y un reporte de caso. De este modo, incluimos en la revisión a 5 trabajos $(14,15,16,17,18)$.

Estudios incluidos: Los 5 estudios incluidos son de diseño retrospectivo y abordan dirigidamente el análisis comparativo entre mantención y retiro del cerclaje $(14,15,16,17,18)$. Dos de ellos realizaron además un estudio caso control de forma paralela $(14,17)$.

Los criterios de inclusión según edad gestacional y las características propias de cada estudio se presentan en la Tabla I. Todas las pacientes se manejaron hospitalizadas, con seguimiento semanal o diario de signos clínicos o de laboratorio de complicaciones infecciosas. Sólo en un estudio se tomaron cultivos vaginales al ingreso (17). Dos estudios incluyeron dentro de su población a pacientes que presentaban RPPM antes de las 24 semanas $(15,16)$. Sin embargo excluyeron de ese grupo a los embarazos que no alcanzaran viabilidad (edad gestacional >24 semanas), o porque la paciente optó por terminación electiva del embarazo. El diagnóstico de RPPM fue similar para la mayoría (historia, examen físico, test de cristalización y test de nitrazina); dos estudios no explicitan mediante qué método diagnóstico se confirmó la RPM $(15,17)$.

Sólo 4 de los 5 trabajos que abordaron el análisis de mantención vs retiro del cerclaje exponen claramente sus criterios de exclusión. Un primer trabajo excluyó los embarazos con RPPM y cerclaje ocurrida antes de las 24 semanas, los embarazos gemelares y a todos los cerclajes terapéuticos o de 
Tabla I

CARACTERÍSTICAS DE LOS ESTUDIOS

\begin{tabular}{|c|c|c|c|c|c|c|c|c|}
\hline Estudio & $\begin{array}{l}\text { Tipo de } \\
\text { estudio }\end{array}$ & Años & $\begin{array}{l}\text { Pacientes } \\
(\mathrm{n})^{\star}\end{array}$ & $\begin{array}{l}\text { EG RPPM } \\
\text { (semanas) }\end{array}$ & $\begin{array}{l}\text { Diagnóstico } \\
\text { RPPM }\end{array}$ & $\begin{array}{l}\text { Tipo de } \\
\text { embarazo }\end{array}$ & $\begin{array}{l}\text { Indicación } \\
\text { de cerclaje }\end{array}$ & $\begin{array}{l}\text { Tipo de } \\
\text { cerclaje }\end{array}$ \\
\hline McElrath $^{14}$ & $\begin{array}{l}\text { Retrospectivo } \\
\text { y caso control }\end{array}$ & $1985-1997$ & 81 & $24,0-34,9$ & $\begin{array}{c}\text { TC y } \\
\text { Nitrazina }\end{array}$ & Único & $\begin{array}{l}\text { Electivo, } \\
\text { emergencia }\end{array}$ & $\begin{array}{l}\text { McDonald } \\
\text { y Shirodkar }\end{array}$ \\
\hline Jenkins $^{15}$ & Retrospectivo & 1990-1999 & 62 & $18-34$ & NC & $\begin{array}{l}\text { Único o } \\
\text { Múltiple }\end{array}$ & $\begin{array}{l}\text { Electivo, } \\
\text { emergencia }\end{array}$ & $\begin{array}{l}\text { McDonald } \\
\text { y Shirodkar }\end{array}$ \\
\hline Kominiarek $^{16}$ & Retrospectivo & $1995-2003$ & 23 & $\leq 32$ & $\begin{array}{c}\text { TC y } \\
\text { Nitrazina }\end{array}$ & $\mathrm{NC}$ & $\begin{array}{l}50 \% \text { de } \\
\text { emergencia }\end{array}$ & McDonald \\
\hline Ludmir $^{17}$ & $\begin{array}{l}\text { Retrospectivo } \\
\text { y caso control }\end{array}$ & $1979-1991$ & 30 & $24-32$ & NC & Único & Electivo & McDonald \\
\hline Kuhn $^{18}$ & Retrospectivo & $1969-1974$ & 69 & NC & NC & $\mathrm{NC}$ & $\begin{array}{l}\text { Electivo, } \\
\text { emergencia }\end{array}$ & $\begin{array}{l}\text { McDonald } \\
\text { y Shirodkar }\end{array}$ \\
\hline
\end{tabular}

$\mathrm{EG}=$ Edad gestacional. RPPM = Rotura prematura pretérmino de membranas. ${ }^{*}$ Pacientes con cerclaje y RPPM. ** Resto no determinado. NC = No Consignado. TC = Test de cristalización.

emergencia (17). La corioamnionitis clínica, el trabajo de parto activo y el estado fetal no tranquilizador al momento de ocurrida la RPPM fueron criterios de exclusión coincidentes para los 3 estudios restantes $(14,15,16)$. Uno de ellos (14) excluyó además a las pacientes que tuvieran su parto antes de $48 \mathrm{hrs}$ de ocurrida la RPPM y otro (15) excluyó a las pacientes con RPPM ocurrida antes de las 24 semanas que optaran electivamente por la terminación del embarazo o que no alcanzaran la viabilidad fetal.

La determinación de mantener o retirar el cerclaje estuvo siempre bajo el criterio del médico tratante: de inmediato luego de la RPPM, o diferido en algún momento de la evolución en las pacientes en las que se mantuvo el cerclaje inicialmente. Habitualmente el retiro del cerclaje fue frente a la ocurrencia de corioamnionitis clínica o inicio franco del trabajo de parto $(14,15,16)$.

Resultados maternos: Dentro de los resultados maternos (Tabla II) el más estudiado fue la incidencia de corioamnionitis clínica. Todos los trabajos reportaron su incidencia; sólo uno describe la incidencia de corioamnionitis clínica y endometritis posparto como un conjunto llamado morbilidad infecciosa materna (15).

Un trabajo encontró mayor incidencia de corioamnionitis clínica en embarazos menores de 28 semanas con cerclaje que presentaron RPPM cuando se mantuvo el cerclaje vs cuando se retiró $(75 \%$ vs $31 \%$ ) (18). Diferencia que no se observó en embarazos con cerclaje en que la RPPM ocurrió pasadas las 28 semanas. Los otros 4 estudios no lograron mostrar diferencias en la incidencia de corioamnionitis clínica $(14,15,16,17)$.

Tabla II

\section{RESULTADOS MATERNOS DE LA COMPARACIÓN ENTRE MANTENER EL CERCLAJE VERSUS RETIRO EN RPPM BAJO 34 SEMANAS}

\begin{tabular}{|c|c|c|c|c|c|c|}
\hline \multirow[b]{2}{*}{ Estudios } & \multicolumn{2}{|c|}{ Corioamnionitis clínica } & \multicolumn{2}{|c|}{ Corioamnionitis histológica } & \multicolumn{2}{|c|}{ Endometritis } \\
\hline & $\begin{array}{c}\text { Mantención } \\
\text { cerclaje \% }\end{array}$ & $\begin{array}{l}\text { Retiro cerclaje } \\
\quad \% \text { (valor } \mathrm{p})\end{array}$ & $\begin{array}{l}\text { Mantención } \\
\text { cerclaje \% }\end{array}$ & $\begin{array}{l}\text { Retiro cerclaje } \\
\% \text { (valor } p)\end{array}$ & $\begin{array}{c}\text { Mantención } \\
\text { cerclaje \% }\end{array}$ & $\begin{array}{l}\text { Retiro cerclaje } \\
\quad \% \text { (valor } p)\end{array}$ \\
\hline McElrath ${ }^{14}$ & 25 & 10 (NS) & 91 & 96 (NS) & 10 & 10 (NS) \\
\hline Jenkins $^{15}$ & $\mathrm{NC}$ & $\mathrm{NC}$ & NC & NC & $\mathrm{NC}$ & NC \\
\hline Kominiarek $^{16}$ & 53 & 50 (NS) & NC & NC & 29,4 & 0 (NS) \\
\hline Ludmir $^{17}$ & 70 & 30 (NS) & NC & NC & $\mathrm{NC}$ & NC \\
\hline Kuhn $^{18}$ & NC & NC & NC & NC & NC & NC \\
\hline
\end{tabular}

$\mathrm{RPPM}=$ Rotura prematura pretérmino de membranas. $\mathrm{NC}=$ No consignado. $\mathrm{NS}=$ No significativo. 
Para las referencias que pudimos metaanalizar se evidenció un aumento en la incidencia de corioamnionitis clínica en los embarazos con cerclaje y RPPM antes de las 34 semanas cuando se mantuvo el cerclaje ( $43 \%$ vs $22 \%$; OR 2,7 ; IC $95 \%$ : $1,46-$ $5,01)(14,16,17,18)$.

Resultados perinatales: La latencia al parto fue el resultado de mayor interés de los estudios. Como se puede ver en la Tabla III, no hubo diferencias significativas de latencia al parto cuando se analizó cada estudio por separado, a excepción de uno que mostró un aumento de la latencia al parto, aumento de la latencia $>48$ horas $y>7$ días luego de ocurrida la RPPM (15). Este trabajo realizó además un subanálisis para embarazos $<28$ semanas con cerclaje que presentaran RPPM, y observaron que la mantención del cerclaje se asociaba a un aumento de la latencia al parto y de la latencia $>7$ días, no así en la latencia $>48$ horas. Vieron además que en este subgrupo de pacientes la mantención del cerclaje se asoció a un mayor peso de nacimiento.

Otro estudio, pese a no encontrar diferencias, demostró que un $30 \%$ de las pacientes había tenido su parto antes de 24 horas posterior al retiro del cerclaje comparado con un $0 \%$ en las mujeres que se mantuvo el cerclaje $(p<0,001)(17)$, y que hasta un $50 \%$ de estas pacientes había tenido su parto antes de las 48 horas una vez retirado el cerclaje (17).

El metaanálisis que efectuamos de estos dos estudios demostró un aumento de la latencia $>48$ horas luego de ocurrida la RPPM en embarazos con cerclaje $<34$ semanas ( $94 \%$ vs $53 \%$; OR 14,85 ; IC95\%: 3,25-67,83) $(15,17)$.

Resultados neonatales: En general la mayoría de los estudios midió como resultado neonatal principal, la incidencia de sepsis neonatal y mortalidad neonatal $(14,15,16)$ (Tabla IV), excepto un estudio que describió solamente la incidencia de muerte fetal intrauterina y de mortalidad neonatal (17).

Ninguno de los estudios logró demostrar mayor incidencia de morbilidad neonatal cuando se comparó mantener vs retirar el cerclaje en relación a: síndrome de distrés respiratorio, enterocolitis necrotizante, hemorragia intraventricular, sepsis neonatal o necesidad de ventilación mecánica.

La incidencia de mortalidad neonatal de los estudios fluctúa entre un $8-70 \%$ en pacientes que se mantiene el cerclaje vs un $7-17 \%$ en las que se retiró el mismo $(14,15,16,17)$. Diferencia que no es significativa cuando se analiza en cada trabajo por separado $(14,15,16)$. Uno de los trabajos reportó que de un total de 7 muertes neonatales, 6 ocurrieron en el grupo de mantención del cerclaje, y todas en embarazos menores de 26 semanas (16). Esta misma publicación reportó a su vez que 3 de esas 6 muertes neonatales ocurrieron producto de sepsis neonatal. Otro trabajo en su análisis de subgrupo reportó que todas las muertes neonatales y todos los recién nacidos con diagnóstico de sepsis neonatal ocurrieron en embarazos <28 semanas (15). El $70 \%$ de las muertes neonatales fueron producto de sepsis neonatal $(p<0,001)(15)$.

Tabla III

\section{RESULTADOS PERINATALES DE LA COMPARACIÓN ENTRE MANTENER EL CERCLAJE VERSUS} RETIRO EN RPPM BAJO 34 SEMANAS

\begin{tabular}{|c|c|c|c|c|c|c|c|c|}
\hline \multirow[b]{2}{*}{ Estudios } & \multicolumn{2}{|c|}{$\begin{array}{l}\text { EG RPPM promedio } \\
\text { (semanas) }\end{array}$} & \multicolumn{2}{|c|}{$\begin{array}{l}\text { EG promedio al parto } \\
\text { (semanas) }\end{array}$} & \multicolumn{2}{|c|}{$\begin{array}{l}\text { Latencia al parto } \\
\text { (días) }\end{array}$} & \multicolumn{2}{|c|}{$\begin{array}{l}\text { Peso de nacimiento } \\
\text { promedio }(\mathrm{g})\end{array}$} \\
\hline & $\begin{array}{l}\text { Mantención } \\
\text { cerclaje \% }\end{array}$ & $\begin{array}{c}\text { Retiro } \\
\text { cerclaje } \\
\% \text { (valor p) }\end{array}$ & $\begin{array}{l}\text { Mantención } \\
\text { cerclaje \% }\end{array}$ & $\begin{array}{c}\text { Retiro } \\
\text { cerclaje } \\
\% \text { (valor } p \text { ) }\end{array}$ & $\begin{array}{l}\text { Mantención } \\
\text { cerclaje \% }\end{array}$ & $\begin{array}{c}\text { Retiro } \\
\text { cerclaje } \\
\% \text { (valor } p \text { ) }\end{array}$ & $\begin{array}{c}\text { Mantención } \\
\text { cerclaje }\end{array}$ & $\begin{array}{l}\text { Retiro } \\
\text { cerclaje } \\
\text { (valor } \mathrm{p})\end{array}$ \\
\hline McElrath ${ }^{14}$ & 28,6 & 28,9 (NS) & 29,7 & 30,1 (NS) & 3 & 4 (NS) & 1450 & 1425 (NS) \\
\hline Jenkins $^{15}$ & 27,7 & 28,0 (NS) & 29,1 & 28,8 (NS) & 10,1 & 4,9 * & 1646 & 1506 (NS) \\
\hline Kominiarek $^{16}$ & 26,1 & 29,3 (NS) & 27,8 & 30,4 (NS) & 12 & 6,3 (NS) & 1035 & 1464 (NS) \\
\hline Ludmir $^{17}$ & 27,7 & 28,6 (NS) & NC & NC & 11 & 5 (NS) & 1397 & 1329 (NS) \\
\hline Kuhn $^{18}$ & NC & NC & NC & NC & 4,3 & $1,27^{*}$ & $\mathrm{NC}$ & NC \\
\hline
\end{tabular}

RPPM = Rotura prematura pretérmino de membranas. $E G=$ Edad gestacional. $\mathrm{NC}=$ No consignado .

NS $=$ No significativo. ${ }^{*} p<0,001$ 
Tabla IV

RESULTADOS NEONATALES DE LA COMPARACIÓN ENTRE MANTENER EL CERCLAJE VERSUS RETIRO EN RPPM BAJO 34 SEMANAS

\begin{tabular}{|c|c|c|c|c|c|c|c|}
\hline Estudios & $\begin{array}{c}\text { MFIU } \\
\text { Mant/Retiro } \\
\text { cerclaje } \\
\text { (valor p) }\end{array}$ & $\begin{array}{c}\text { MN } \\
\text { Mant/Retiro } \\
\text { cerclaje } \\
\text { (valor p) }\end{array}$ & $\begin{array}{c}\text { SDR } \\
\text { Mant/Retiro } \\
\text { cerclaje } \\
\text { (valor p) }\end{array}$ & $\begin{array}{c}\text { ECN } \\
\text { Mant/Retiro } \\
\text { cerclaje } \\
\text { (valor p) }\end{array}$ & $\begin{array}{c}\text { HIV } \\
\text { Mant/Retiro } \\
\text { cerclaje } \\
\text { (valor p) }\end{array}$ & $\begin{array}{c}\text { SN } \\
\text { Mant/Retiro } \\
\text { cerclaje } \\
\text { (valor p) }\end{array}$ & $\begin{array}{c}\text { VM } \\
\text { Mant/Retiro } \\
\text { cerclaje } \\
\text { (valor p) }\end{array}$ \\
\hline McElrath ${ }^{14}$ & $\mathrm{NC}$ & 0,845 & 0,576 & 0,710 & 0,329 & 0,845 & 0,993 \\
\hline Jenkins $^{15}$ & $\mathrm{NC}$ & 0,64 & 0,45 & $\mathrm{NC}$ & $\mathrm{NC}$ & 0,29 & $\mathrm{NC}$ \\
\hline Kominiarek $^{16}$ & $\mathrm{NC}$ & 0,55 & $\mathrm{NC}$ & NC & $\mathrm{NC}$ & 0,53 & NC \\
\hline Ludmir $^{17}$ & $>0,05$ & $<0,001$ & NC & $\mathrm{NC}$ & NC & $\mathrm{NC}$ & NC \\
\hline Kuhn $^{18}$ & $\mathrm{NC}$ & NC & NC & NC & NC & NC & NC \\
\hline
\end{tabular}

RPPM = Rotura prematura pretérmino de membranas. Mant = Mantención. MFIU = Muerte fetal in útero. MN = Mortalidad neonatal. SDR = Síndrome de distrés respiratorio. ECN = Enterocolitis necrotizante. HIV = Hemorragia intraventricular. $\mathrm{SN}=$ Sepsis neonatal. VM = Ventilación mecánica. $\mathrm{NC}=$ No consignado.

El metaanálisis de los resultados de los 4 trabajos para las mujeres en que se mantuvo el cerclaje encontramos lo siguiente:

a. No hubo aumento en la incidencia de mortalidad neonatal en embarazos con cerclaje que presentaron RPPM antes de las 28 semanas $(15,16)(22 \%$ vs $21 \%$; OR 1,11; IC95\%: 0,3-4,05) ni antes de las 34 semanas $(14,15,16,17)$ (17\% vs $9,6 \%$; OR 1,95 ; IC95\%: 0,83-4,6).

b. Aumento de la mortalidad neonatal por sepsis en embarazos con cerclaje y RPPM antes de las 34 semanas $(16,17)$ (32\% vs $4 \%$; OR 11,76 ; IC $95 \%$ : 1,35-102,86).

Revisión por intervención: Como se puede ver en la
Tabla $\mathrm{V}$, existen variaciones en cuanto a las intervenciones realizadas a estas pacientes. En sólo 2 estudios se trató al $100 \%$ de las pacientes con Eritromicina al menos $(9,16)$. El resto trató a algunas de sus pacientes o simplemente no las trató, y de las que recibieron antibióticos no especifican qué régimen ni por cuánto tiempo. La dosis de corticoides fue la misma en todos los trabajos y equivalente a la que se utiliza en la actualidad, sin embargo, no todas las pacientes fueron tratadas (17). En 3 de 4 estudios se usó tocolisis con sulfato de magnesio, al menos por 48 horas, para completar la dosis de corticoides $(14,15,16)$. El uso de amniocentesis no se describe como de rutina entre las publicaciones.

Tabla V

INTERVENCIONES CON DIAGNÓSTICO DE RPPM

\begin{tabular}{llcccc}
\hline Estudio & ¿Retiro del cerclaje? & AMCT & $\begin{array}{c}\text { Antibióticos } \\
(\%)\end{array}$ & $\begin{array}{c}\text { Corticoides }^{* *} \\
\text { (\%) }\end{array}$ & $\begin{array}{c}\text { Tocolisis }^{* * *} \\
(\%)\end{array}$ \\
\hline McElrath $^{14}$ & Decisión del tratante & NC & 35 & 80 & 18 \\
Jenkins $^{15}$ & Inmediato v/s diferido & NC & 89 & 71 & 10 \\
Kominiarek $^{16}$ & Decisión del tratante & NC & 100 & 100 & 65 \\
Ludmir $^{17}$ & Decisión del tratante & No & 0 & 0 & 0 \\
Kuhn $^{18}$ & NC & NC & Si (\% NC) & NC & NC \\
\hline
\end{tabular}

RPPM = Rotura prematura pretérmino de membranas. AMCT = Amniocentesis. *Antibióticos = Monoterapia o biterapia; ${ }^{\star \star}$ Esquema 2 dosis c/24 h de $12 \mathrm{mg}$ IM betametasona. ${ }^{* \star \star}$ Sulfato de magnesio. NC = No consignado. 


\section{DISCUSIÓN}

Encontramos 5 publicaciones que comparan la mantención y el retiro del cerclaje en mujeres embarazadas con cerclaje que presentan una RPPM antes de las 34 semanas. En estas pacientes la mantención del cerclaje se asocia a un aumento de la corioamnionitis clínica, a un aumento de la mortalidad neonatal por sepsis y a un aumento de la latencia al parto $>48$ horas luego de ocurrida la RPPM.

Si bien la incidencia de corioamnionitis clínica no mostró diferencias en cada estudio por separado, el metanálisis de los resultados mostró que la incidencia casi alcanza a triplicarse cuando se mantuvo el cerclaje in situ luego de ocurrida la RPPM (43\% vs $22 \%$; OR 2,7$)$.

La mortalidad neonatal atribuible a sepsis fue 10 veces mayor en embarazos en los que se mantuvo el cerclaje. Este es un punto importante a considerar cuando se discute morbimortalidad neonatal dado que en estos pacientes el resultado neonatal final va a depender directamente de la edad gestacional al parto, por los riesgos de prematurez y de la morbilidad asociada a la prematurez extrema. Al analizar en conjunto los resultados de latencia al parto mayor a 48 horas luego de ocurrida la RPPM $(15,17)$, observamos que la latencia al parto aumentó significativamente. Esto sin duda es relevante pues permite efectuar la inducción de madurez pulmonar con corticoides, tratamiento que reduce significativamente la morbimortalidad perinatal de recién nacidos menores a 34 semanas.

Los datos obtenidos de estudios incluidos en nuestra revisión, no son completamente representativos de nuestra situación actual, debido a que la mayoría de las intervenciones realizadas durante el embarazo han ido cambiando. Hoy en día el uso de corticoides y de antibióticos profilácticos es indiscutible para el manejo de RPPM de pretérmino (19). Debido a que varios de estos trabajos preceden a la era del ORACLE (20) es que hay inconsistencia en cuanto al tratamiento antibiótico administrado en cada trabajo. Lo mismo sucedió con la tocolisis; si bien en casos de prematurez extrema se podría considerarla como medida última para lograr completar el curso de corticoides, la evidencia actual la contraindica dado su mayor asociación con corioamnionitis (21).

Existen 2 revisiones sistemáticas en la literatura que analizan el tema de nuestra revisión. Una de ellas, mediante una metodología de análisis muy similar a la nuestra, evaluó los resultados maternos y perinatales, con el fin de comparar los beneficios 0 riesgos asociados a la mantención o retiro del cer- claje. Concluyen que con la literatura disponible es difícil determinar la mejor conducta, por lo que sugieren un manejo individualizado para cada paciente balanceando riesgos de infección, prematurez, etc. (12).

La segunda revisión, además de revisar los resultados de cada trabajo por separado realizó un metanálisis (13). Incluyeron dentro de su análisis una publicación que nosotros descartamos (11) pues compara pacientes con RPPM con o sin cerclaje. Esta revisión (13) concluye que el beneficio de mantener el cerclaje, para prolongar la latencia y disminuir las complicaciones relacionadas a la prematurez, debe ser cautamente balanceado a los riesgos asociados a la morbilidad materna y la morbimortalidad neonatal, y por eso propusieron un algoritmo de manejo para retirar el cerclaje en base a la edad gestacional.

La evidencia expuesta demuestra que la mantención del cerclaje en pacientes con RPPM aumenta la latencia al parto, pero lo hace a expensas de un aumento en la morbilidad infecciosa materna y aumento en la morbimortalidad neonatal por sepsis $(14,15,16,17,18)$. Si bien la mortalidad neonatal en globo no aumenta, llama la atención que en al menos 2 trabajos el $100 \%$ de la mortalidad neonatal ocurrió en embarazos con cerclaje y RPPM antes de las 28 semanas $(15,16)$. Lo anterior refuerza la importancia de la prematurez extrema y la morbilidad asociada a ella sobre el resultado neonatal.

\section{CONCLUSIÓN}

De los hallazgos expuestos y en coincidencia con las recomendaciones de una de las revisiones sistemáticas mencionadas (13), creemos que la mejor conducta en mujeres con cerclaje y RPPM es mantener el cerclaje in situ por 48 horas, en la medida que sea posible, con el objetivo de lograr completar el curso de corticoides y luego retirarlo. Una vez retirado el cerclaje se procede al manejo usual de un embarazo con RPPM antes de las 34 semanas. En nuestro centro, si la edad gestacional es menor a 32 semanas, el manejo es conservador: se indica un curso de 10 días de antibióticos por vía oral (eritromicina y ampicilina) y vigilancia estricta por aparición de signos de infección (clínicos y de laboratorio); intentando llegar a las 32 semanas. Si la RPPM ocurre después de las 32 semanas, el manejo es activo, con AMCT para descartar invasión microbiana de la cavidad amniótica y certificar madurez pulmonar. Si se descarta madurez e infección ovular, se procede a la interrupción del embarazo una vez completada la inducción de madurez pulmonar. 


\section{REFERENCIAS}

1. ACOG. Management of preterm labor. Practice Bulletin No. 127. Obstet Gynecol 2012;119:1308-17.

2. MINSAL. Prevención del parto prematuro. Guía Clínica 2010;1-53.

3. ACOG. Cervical Insufficiency. Practice Bulletin No. 48. Obstet Gynecol 2003;102:1091-9.

4. Owen J, Hankins G, lams JD, Berghella V, Sheffield JS, Perez-DElboy A, et al. Multicenter randomized trial of cerclage for preterm birth prevention in high-risk women with shortened midtrimester cervical length. Am J Obstet Gynecol 2009;201:375.e1-8.

5. Berghella V, Rafael T, Szychowski J, Rust O, Owen J. Cerclage for short cervix on ultrasonography in women with singleton gestations and previous preterm birth: meta-analysis of trials using individual patientlevel data. Obstet Gynecol 2011;117:663-71.

6. ACOG. Premature rupture of membranes. Clinical management for obstetrician-gynecologists. Practice Bulletin No. 80. Obstet Gynecol 2007;109:1007-19.

7. Nelson L, Dola T, Tran T, Carter M, Luu H, Dola C. Pregnancy outcomes following placement of elective, urgent and emergent cerclage. J Matern Fetal Neonatal Med 2009;22:269-73.

8. McElrath TF, Norwitz ER, Lieberman ES, Heffner LJ. Perinatal outcome after preterm premature rupture of membranes with in situ cervical cerclage. Am J Obstet Gynecol 2002;187:1147.

9. Laskin MD, Yinon Y, Whittle WL. Preterm premature rupture of membranes in the presence of cerclage: is the risk for intra-uterine infection and adverse neonatal outcome increased? J Matern Fetal Neonatal Med 2012;25(4):424-8.

10. Yeast JD, Garite TR. The role of cervical cerclage in the management of preterm premature rupture of membranes. Am J Obstet Gynecol 1988;158:106-10.

11. Blickstein I, Katz Z, Lancet M, Molgilner BM. The outcome of pregnancies complicated by preterm rup- ture of the membranes with and without cerclage. Int $\mathrm{J}$ Gynecol Obstet 1989;28:237-42.

12. Walsh J, Allen V, Colford D, Allen A. Preterm prelabour rupture of membranes with cervical cerclage: a review of perinatal outcomes with cerclage retention. J Obstet Gynaecol Can 2012;32:448-52.

13. Giraldo-Isaza M, Berghella V. Cervical cerclage and preterm PROM. Clin Obstet Gynecol 2011;54:313-20.

14. McElrath TF, Norwitz ER, Lieberman ES, Heffner LJ. Management of cervical cerclage and preterm premature of the membranes: should the stitch be removed? Am J Obstet Gynecol 2000;183:840-6.

15. Jenkins TM, Berghella V, Shlossman PA, Mclntyre CJ, Maas BD, Pollock MA, Wapner RJ. Timing of cerclage removal after preterm premature rupture of membranes: Maternal and neonatal outcomes. Am J Obstet Gynecol 2000;183:847-52.

16. Kominiarek MA, Kemp A. Perinatal outcome in preterm premature rupture of membranes at $<$ or $=32$ weeks with retained cerclage. J Reprod Med 2006;51:533-8.

17. Ludmir J, Bader T, Chen L, Lindenbaum C, Wong G. Poor perinatal outcome associated with retained cerclage in patients with premature rupture of membranes. Obstet Gynecol 1994;84:823-6.

18. Kuhn R, Pepperell R. Cervical ligation: a review of 242 pregnancies. Aust N Z J Obstet Gynaecol 1977;17:7983.

19. Kenyon S, Boulvain M, Neilson JP. Antibiotics for preterm rupture of membranes. Cochrane Database of Systematic Reviews 2010, Issue 8.

20. Kenyon S, Taylor D, Tarnow-Mordi W. Broad-spectrum antibiotics for preterm, prelabour rupture of fetal membranes: the ORACLE I randomized trial. Lancet 2001;357:979-88.

21. Mackeen AD, Seibel-Seamon J, Grimes-Dennis J, Baxter JK, Berghella V. Tocolytics for preterm premature rupture of membranes. Cochrane Database of Systematic Reviews 2011, Issue 10. 